\title{
PASSAGE DE POISSONS DU RHÔNE PAR LES POMPES D'IRRIGATION DE CAMARGUE : PREMIERS RÉSULTATS.
}

\author{
G. POIZAT, P. CHAUVELON, E. ROSECCHI, A.J. CRIVELLI \\ avec la collaboration technique de P. CONTOURNET
}

Station Biologique de la Tour du Valat, Le Sambuc, 13200 Arles, France.

Reçu le 18 mai 1998

Accepté le 05 octobre 1998
Received 18 May, 1998

Accepted 05 October, 1998

\section{RÉSUMÉ}

Les pompes d'irrigation constituent la principale connexion aquatique entre liîle de Camargue et d'autres milieux d'eau douce. Nous avons suivi le passage de poissons dans 2 sites parmi les 15 stations de pompage du bassin du Fumemorte $\left(68 \mathrm{~km}^{2}\right)$ pendant toute la période rizicole, c'est-à-dire de fin avril à début octobre 1997.

Les poissons peuvent survivre au passage par la pompe. En grande majorité, ce sont des individus de petites tailles $(2-5 \mathrm{~cm})$. Cependant, des individus de grandes tailles (jusqu'à $46 \mathrm{~cm}$ ) sont passés vivants. On estime à 14700 le nombre d'individus entrés en Camargue par la pompe du Sambuc, et à 98000 pour l'ensemble du bassin du Fumemorte. Vingt-quatre espèces ont été capturées par pompage. Les espèces dominantes numériquement sont le gardon, l'anguille, le goujon et la brème bordelière.

Ces résultats sont comparés aux données sur les peuplements d'autres milieux de Camargue, afin de discuter l'importance de l'immigration par pompage dans le tonctionnement des peuplements de poissons de Camargue. Ils suggèrent que (1) le pompage permet l'introduction d'espèces nouvelles en Camargue, (2) les populations de certaines espèces passant par les pompes ne sont maintenues en Camargue que par cette immigration (e.g., hotu, chevaine, barbeau), (3) les poissons utilisant les marais temporaires viennent davantage du réseau de drainage que du réseau d'irrigation (e.g., épinoche, gobie commun, athérine), et (4) certaines espèces pompées ont des populations abondantes en Camargue (e.g., anguille, Pseudorasbora parva, ablette, brèmes), pour celles-ci, l'importance de l'immigration par pompage dans le fonctionnement de leurs populations en Camargue reste difficile à évaluer.

\section{PASSAGE OF RHÔNE RIVER FISH THROUGH IRRIGATION PUMPS IN THE CAMARGUE : FIRST RESULTS.}

\section{ABSTRACT}

Irrigation pumps are the main aquatic connexions between the Isle of Camargue and any other freshwater system. Fish passage was monitored in 2 sites among the 15 pumping stations of the Fumemorte catchment $\left(68 \mathrm{~km}^{2}\right)$ during an entire rice cultivation period, from April to October 1997. 
Fish can survive the passage through the pumps. They were mainly small-sized $(2-5 \mathrm{~cm}$ ). However, large fish (up to $46 \mathrm{~cm}$ ) can pass alive through the pumps. Estimation of fish quantity entering through the Sambuc pump was 14,700 individuals and 98,000 for the whole Fumemorte basin. Twenty four species were caught in our samples. Dominant species were roach, eel, gudgeon and white bream.

The species composition of the pumped fish was compared with the fish assemblages of other habitats in the Camargue, in order to assess the significance of this immigration in the functioning of fish populations in the Fumemorte drainage basin. Pumped fish assemblage was characterized by the relative abundance of Rhone River species such as the nase, the chub, the barbel, the roach, the gudgeon and the shad. A sampling site in an irrigation canal located $10 \mathrm{~km}$ far from the pumping station was shown to bear a fish assemblage which was relatively close to the pumped one. Fish assemblage from another sampling site located in the Fumemorte drainage canal differed from those of the irrigation system by the relative rarity or absence of some Rhone River species such as the nase, the chub or the barbel, and the relative abundance of species of brackish waters which enter the drainage system by the Vaccarès lagoon, such as the three-spine stickleback, the common goby or the sand smelt. In a seasonally-flooded marsh connected occasionally both to the irrigation system and to the drainage system, the fish assemblage appeared to be a subset of the drainage canal assemblage, in which species such as the roach, the gudgeon and the pike-perch were lacking. These results suggest the following roles of immigration through pumping in the functioning of fish populations in the Camargue :(1) pumping allows exotic species to enter the Camargue waters, (2) some of the pumped fish species do not find suitable habitat conditions in the Camargue and their populations in the Camargue are only maintained by immigration, (3) fishes in seasonally-flooded marshes come more from the drainage system than from the irrigation one, and (4) for the pumped fish species having abundant populations in the Camargue, the importance of this immigration in the functioning of their populations in the Camargue remains diffficult to assess.

\section{INTRODUCTION}

Depuis les endiguements du Rhône et la construction de la digue à la mer, à la moitié du XIX siècle, les connexions aquatiques entre l'île de Camargue et l'extérieur sont très limitées. Avec la mer, les seules connexions sont le grau de la Fourcade et le grau de Rousty. Avec le Rhône, les principales connexions sont les pompes d'irrigation et de drainage, les autres connexions sont anecdotiques et ne se rencontrent que sur le Petit Rhône ; il s'agit de prises d'eau gravitaires, consistant en des ouvertures dans la digue contrôlées par des martelières, dont les capacités d'irrigation sont dépendantes du niveau du Rhône, et très limitées en comparaison avec les pompes (CHAUVELON, 1996). Ainsi, pour les espèces strictement d'eau douce, les pompes constituent pratiquement la seule connexion avec l'extérieur de la Camargue.

Dans l'île de Camargue, environ 400 millions de $\mathrm{m}^{3}$ d'eau du Rhône sont pompés annuellement pour l'irrigation de la riziculture, d'avril à octobre (HEURTEAUX, 1994). Cependant, le Vaccarès et les étangs inférieurs n'ont pas la capacité suffisante pour drainer la totalité de ce volume d'eau sans risquer des inondations en cas de fortes pluies automnales. C'est pourquoi $55 \%$ de la surface de l'île de Camargue est poldérisée, c'est-àdire que l'eau de drainage retourne au Rhône par pompage (CHAUVELON et al., 1996 ; CHAUVELON, 1998).

Le bassin du Fumemorte représente $55 \%$ de la surface non-poldérisée, dont l'eau de drainage s'écoule gravitairement dans l'étang du Vaccarès. Le fonctionnement hydrologique et écologique des bassins non-poldérisés est dépendant, vers l'amont, du pompage dans 


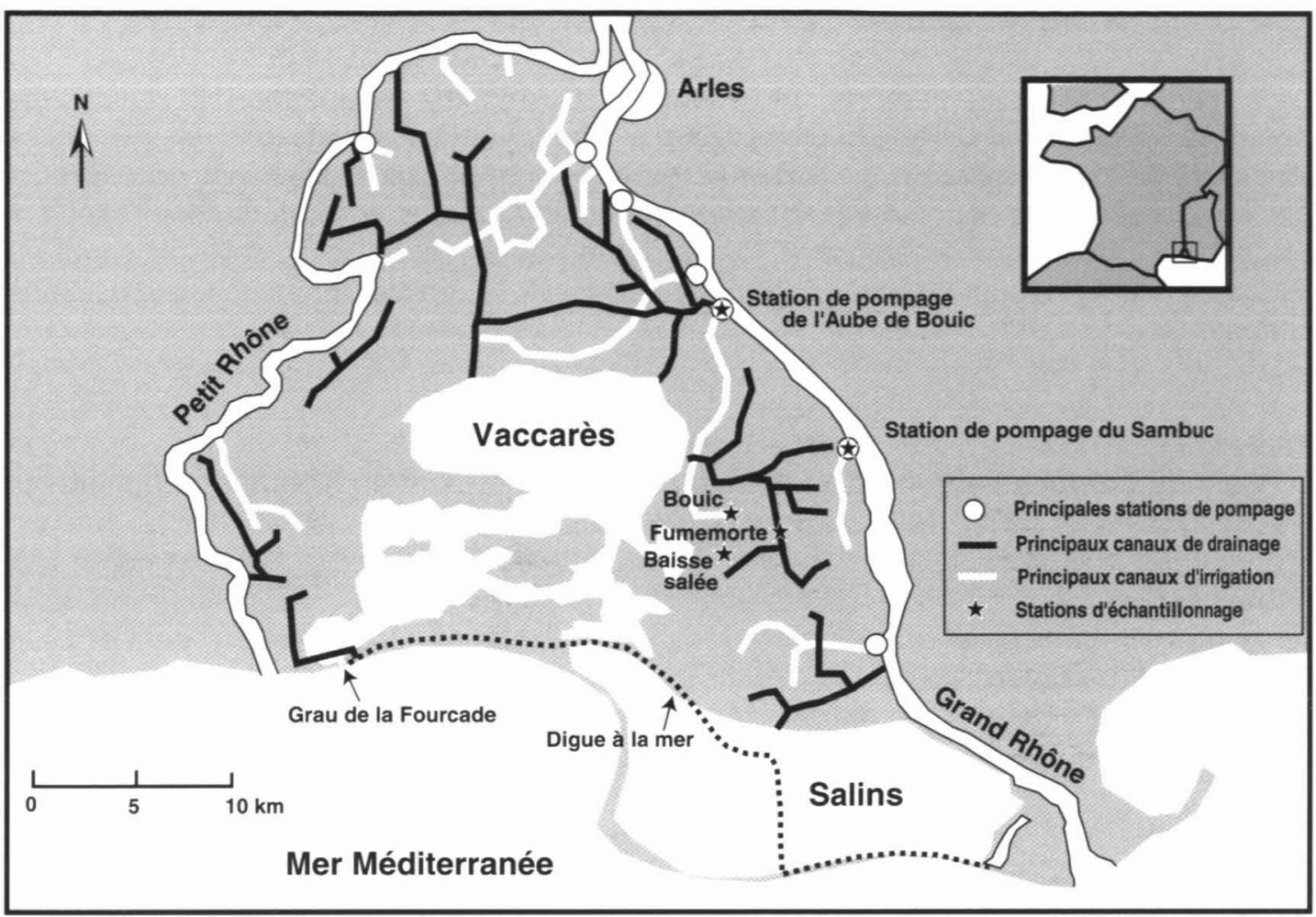

Figure 1

Situation géographique de la zone d'étude en Camargue et localisation des stations d'échantillonnage.

\section{Figure 1}

Geographical situation of the study area in the Camargue and localisation of the sampling sites.

le Grand Rhône pour l'irrigation, et vers l'aval, de l'étang du Vaccarès, des étangs inférieurs et des échanges avec la mer (F.igure 1). A la Station Biologique de la Tour du Valat, ce bassin fait l'objet d'études hydrologiques (CHAUVELON, 1996, 1998 ; CHAUVELON et al., 1996) et ichtyologiques (CRIVELLI; 1981 ; CRIVELLI et BRITTON, 1987 ; CRIVELLI et MESTRE, 1988 ; ROSECCHI et CRIVELLI, 1992, 1995 ; ROSECCHI et al., 1997 ; POIZAT et CRIVELLI, 1997). Des études sont en cours sur les peuplements de poissons du Vaccarès et des étangs inférieurs (E. ROSECCHI, C. PAMPOULIE, A.J. CRIVELLI, données non publiées). BARDIN (1994) a étudié les passages de poissons de la mer vers les étangs inférieurs de Camargue par le grau de la Fourcade, mais on ne dispose pas d'estimation des quantités de poissons passant dans une année. Dans le but d'arriver à une compréhension globale du fonctionnement des populations de poissons en Camargue, il était important d'identifier et de quantifier l'immigration de poissons par les pompes d'irrigation.

Dans cet article, nous présentons les résultats du suivi du passage de poissons par deux stations de pompage pendant la saison rizicole de 1997.-Ces résultats sont comparés aux données dont on dispose sur les peuplements des canaux d'irrigation et de drainage et les marais temporaires du bassin du Fumemorte, afin de discuter l'importance de cette immigration sur le'fonctionnement des populations de poissons en Camargue. 


\section{MATÉRIEL ET MÉTHODES}

Le bassin du Fumemorte, représentant $68 \mathrm{~km}^{2}$, est équipé de 15 stations de pompage (Figure 1). CHAUVELON (1996) a regroupé ces 15 stations en 10 unités fonctionnelles de pompage, car certaines exploitations agricoles disposent de plusieurs stations de pompage pour irriguer leurs terres (Tableau I). Dans cette étude, deux sites ont été choisis : la station de pompage du canal de l'Aube de Bouic et celle du canal du Sambuc, situées respectivement à environ $33 \mathrm{~km}$ et $25 \mathrm{~km}$ de l'embouchure du Grand Rhône.

\section{Tableau I}

Caractéristiques hydrauliques des stations de pompage du bassin du Fumemorte (données de l'année 1994 d'après CHAUVELON, 1996).

\section{Table I}

Hydraulic features of pumping stations in the Fumemorte catchment (data of 1994 after CHAUVELON, 1996).

\begin{tabular}{lcc}
\hline Station de pompage & $\begin{array}{c}\text { débit maximum } \\
(1 / \mathrm{s})\end{array}$ & $\begin{array}{c}\text { estimation du volume } \\
\text { pompé en une année } \\
\text { (millions de m3) }\end{array}$ \\
\hline Aube de Bouic & 2420 & 24,5 \\
Sambuc & 1150 & 10,5 \\
Tourtoulen-Giraud & 1040 & 9,5 \\
Cazeau & 530 & 4,0 \\
Petit Paty & 690 & 4,3 \\
Grand Manusclat & 730 & 7,4 \\
Grifeuille-Peaudure & 720 & 4,1 \\
Chartrouse & 560 & 2,8 \\
Louisiane & 330 & 1,5 \\
Amérique & 365 & 1,6 \\
\hline & & \\
Total & 8535 & 70,1 \\
\hline
\end{tabular}

Du point de vue hydraulique, les stations de pompage peuvent être caractérisées par leur débit instantané et par le volume d'eau pompé sur toute une saison rizicole (tableau I). Toutes les pompes des stations sont de type centrifuge. La station de pompage de l'Aube de Bouic est équipée de deux pompes FLYGT de $1000 \mathrm{l} / \mathrm{s}$ chacune, d'une pompe RATEAU de $1000 \mathrm{l} / \mathrm{s}$ et de deux pompes RATEAU de $400 \mathrm{l} / \mathrm{s}$ chacune. Ces pompes ne fonctionnent jamais toutes en même temps. La station de pompage du Sambuc est équipée de deux pompes FLYGT de $500 \mathrm{l} / \mathrm{s}$ chacune et d'une pompe RATEAU de $200 \mathrm{l} / \mathrm{s}$. Leurs moteurs électriques sont équipés de compteurs horaires permettant, a posteriori, de calculer le volume pompé à partir du nombre d'heures de fonctionnement et du débit de la pompe (CHAUVELON, 1996). Les informations sur les caractéristiques de la prise d'eau sont difficiles à obtenir car les installations sont anciennes. Les prises d'eau sont généralement équipées d'une grille de $5 \mathrm{~cm}$ de vide de maille afin d'éviter de pomper de trop gros débris.

Au Sambuc, le débit de la pompe et la vitesse du courant dans le canal (comprise entre 0,4 et $0,6 \mathrm{~m} / \mathrm{s} ; \mathrm{P}$. CHAUVELON, données non publiées) permettent d'utiliser un piège qui filtre tout le volume pompé. A l'Aube de Bouic, le débit et la vitesse du courant dans le canal (comprise entre 0,8 et $1 \mathrm{~m} / \mathrm{s} ; P$. CHAUVELON, données non publièes) sont trop forts 
pour pouvoir relever un piège comme celui du Sambuc. De ce fait, à l'Aube de Bouic, nous avons utilisé un piège ne filtrant qu'environ 1/5 du volume pompé (ce ratio est approximatif car il dépend de la hauteur d'eau dans le canal). Les pièges sont constitués de verveux (appelés localement " ganguis ") de $5 \mathrm{~mm}$ de vide de maille et dont les anneaux terminaux sont doublés d'une moustiquaire de moins d' $1 \mathrm{~mm}$ de vide de maille. Les pièges sont fixés à un cadre métallique de la taille du canal bétonné (environ $1,2 \mathrm{~m} \times 1 \mathrm{~m}$ ). A l'Aube de Bouic, les ailes du verveux ont été supprimées et les anneaux du piège sont.accrochés.au .cadre . par quatre câbles.

Les pièges sont posés consécutivement un jour et une nuit, deux fois par semaine, et ceci, trois semaines par mois pendant toute la période rizicole, c'est-à-dire de fin avril à début octobre. La taille des poissons est mesurée par la longueur à la fourche.

Pour estimer les quantités de poissons passant par une pompe au cours de la période rizicole, les effectifs de poissons passant les jours non échantillonnés sont estimés par la moyenne du jour échantillonné précédent et du jour échantillonné suivant. En raison du sous-échantillonnage approximatif à la station de l'Aube de Bouic, seules les données du Sambuc feront l'objet d'une estimation quantitative.

L'estimation des quantités de poissons pompés sur l'ensemble du bassin du Fumemorte au cours de la période rizicole repose sur les données du Sambuc. Pour chacune des 14 autres stations de pompage, les quantités de poissons sont grossièrement estimées par une règle de trois, connaissant la quantité de poissons pompée au Sambuc, le volume d'eau pompé au Sambuc, et le volume d'eau pompé dans la station de pompage à estimer (tableau I).

Les résultats des échantillonnages aux stations de pompage du Sambuc et de l'Aube de Bouic sont comparés à ceux des trois stations suivantes, représentant trois compartiments de l'hydrosystème du bassin du Fumemorte (Figure 1) :

- le canal d'irrigation de l'Aube de Bouic à $10 \mathrm{~km}$ en aval de la pompe,

- le canal de drainage du Fumemorte à $6 \mathrm{~km}$ en amont de l'embouchure,

- le marais de la Baisse salée, qui est connecté à la fois au canal de l'Aube de Bouic et au canal du Fumemorte.

Les peuplements de poissons du canal d'irrigation de l'Aube de Bouic et du canal de drainage du Fumemorte sont échantillonnés mensuellement de novembre 1996 à octobre 1997, à l'aide d'une paire de verveux de $5 \mathrm{~mm}$ de vide de maille relevés quotidiennement pendant 4 jours consécutifs (ROSECCHI et al., 1997 ; POIZAT et CRIVELLI, 1997). Le marais de la Baisse salée a été échantillonné au moment de son assèchement annuel en mai 1996, au moyen de quatre capétchades (engin constitué d'une paradière, i.e. un filetbarrage rectiligne, qui guide les poissons jusqu'à trois verveux de $5 \mathrm{~mm}$ de vide de maille) relevées quotidiennement pendant 10 jours consécutifs. La gambusie n'est pas correctement échantillonnée dans le marais de la Baisse salée en raison de sa distribution spatiale dans les zones peu profondes, alors que les capétchades sont posées dans les zones les plus profondes. En conséquence, nous avons éliminé cette espèce de notre analyse comparative.

Le tableau des effectifs des espèces dans les captures des quatre stations (en cumulant les deux stations de pompage) a été soumis à une analyse factorielle des correspondances (AFC, THIOULOUSE et al., 1997), de manière à ordonner les stations d'après leurs ressemblances faunistiques et à ordonner les espèces d'après leurs abondances relatives dans les différentes stations. En utilisant la distance du $\chi^{2}$, l'AFC permet d'analyser la distribution des captures des différentes espèces dans les différentes stations bien que l'effort d'échantillonnage n'y soit pas constant. 

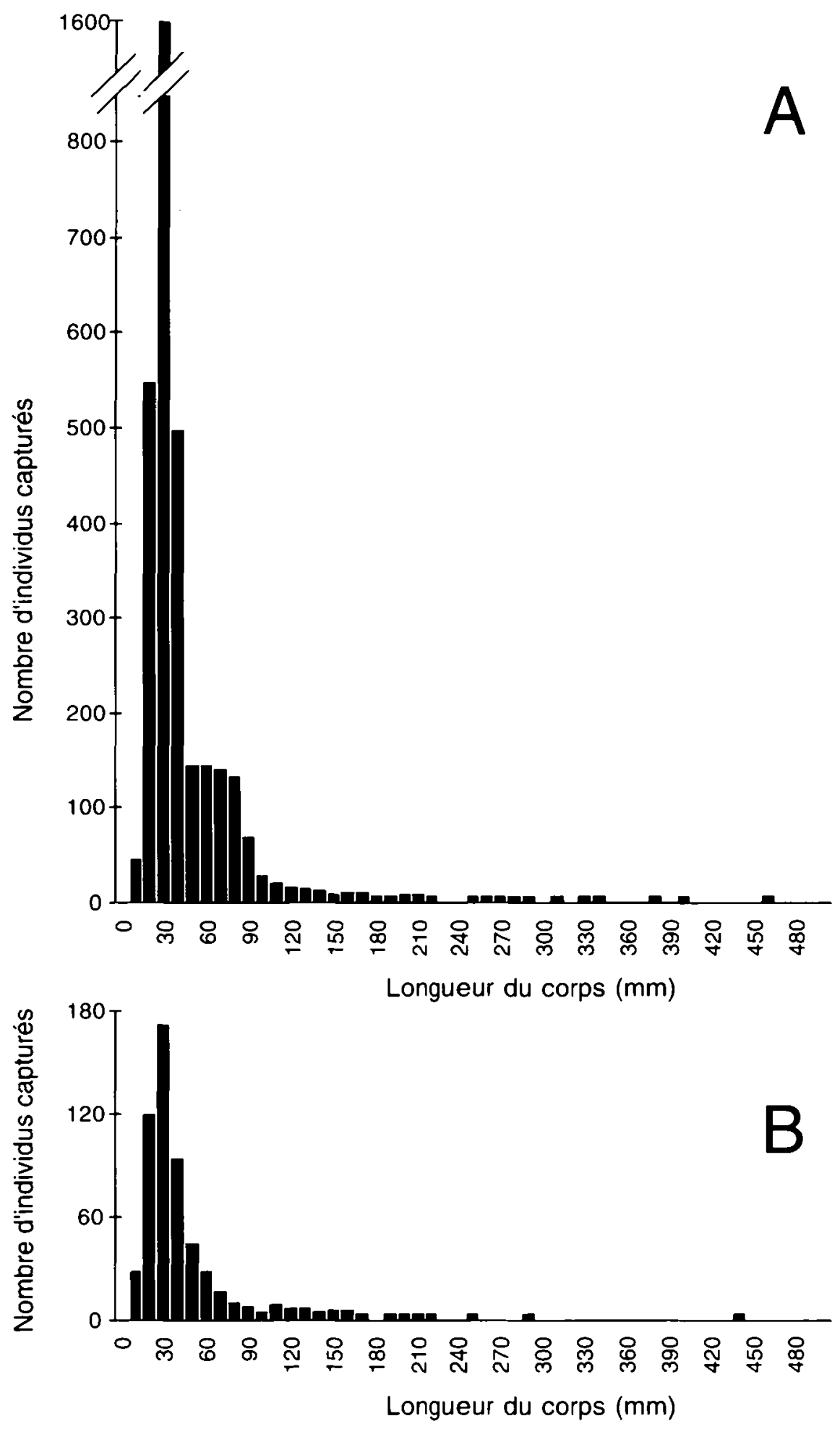

Figure 2

Distribution des tailles de poissons passant par les pompes du Sambuc (A) et de l'Aube de Bouic (B). La taille est mesurée par la longueur à la fourche.

\section{Figure 2}

Size distribution of fish passing through the pumps of Le Sambuc (A) and l'Aube de Bouic (B). Body size is measured by fork length. 


\section{RÉSULTATS}

Effectivement, des poissons passent par les pompes. Les poissons capturés ne montraient pas de blessures particulières qui auraient pu être attribuées aux chocs mécaniques liés au passage dans la pompe. Seul un individu a été capturé coupé en deux; c'était un gardon dont la taille devait être de $33 \mathrm{~cm}$. De nombreux individus de petite taille ont été trouvés morts dans le piège, en particulier lorsque les effectifs capturés étaient très importants. En dehors de ces cas, les poissons étaient trouvés vivants dans les pièges.

Les poissons entrant par les pompes sont principalement des individus de petites tailles, entre 2 et $5 \mathrm{~cm}$ (Figure 2). Cependant, des individus de grandes tailles (46 cm pour l'anguille, $34 \mathrm{~cm}$ pour les muges, $29 \mathrm{~cm}$ pour la brème bordelière) sont passés vivants.

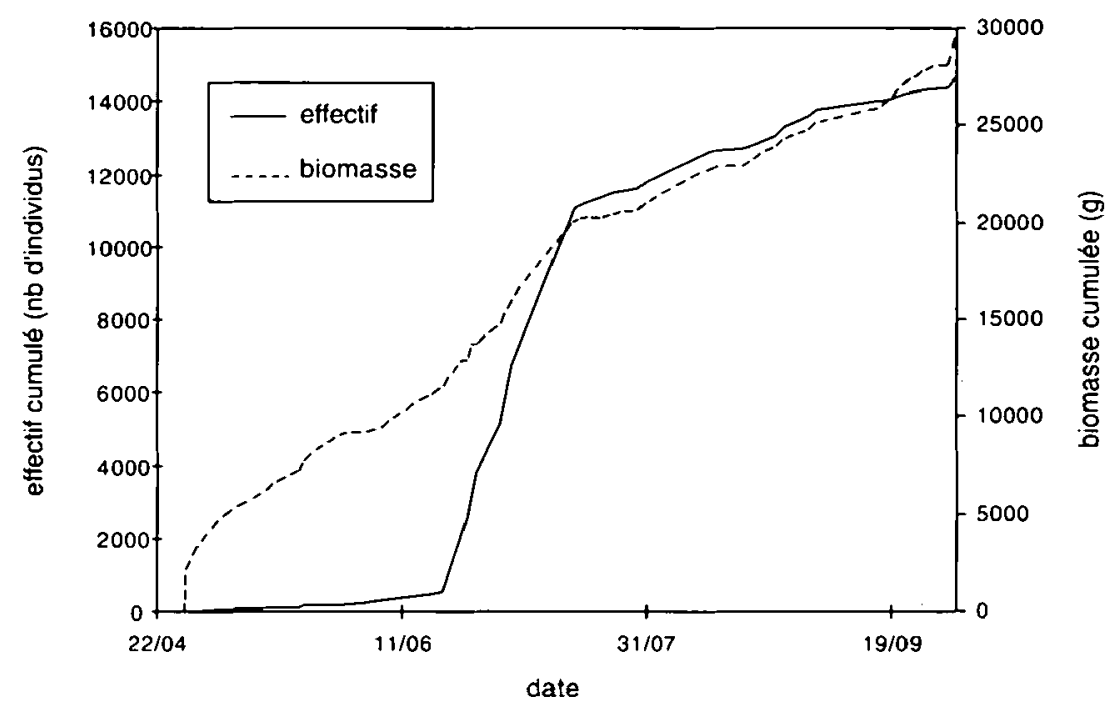

\section{Figure 3}

Estimation de l'effectif et de la biomasse des poissons passant par la pompe du Sambuc pendant la période rizicole 1997.

\section{Figure 3}

Estimation of number and biomass of fish passing through the Sambuc pump during the 1997 rice cultivation period.

On estime à 14700 le nombre d'individus entrés en Camargue par la pompe du Sambuc, représentant une biomasse de $29,7 \mathrm{~kg}$. L'évolution de l'effectif cumulé au cours du temps (Figure 3) montre que la plus grande partie des individus passe entre mi-juin et mijuillet. Ces individus sont principalement des juvéniles de Cyprinidés (surtout de gardon), ce qui explique qu'ils ne représentent pas une part aussi importante en biomasse.

L'estimation globale du nombre de poissons pompés pendant la saison rizicole de 1997 dans le bassin du Fumemorte, calculée en extrapolant à partir des données recueillies à la station de pompage du Sambuc, est de 98000 individus.

Pour les petits cyprinidés retrouvés en mauvais état dans les pièges, la détermination spécifique était impossible. Leur effectif a été réparti d'après les abondances relatives des espèces auxquelles leur faciès pouvait correspondre (gardon, chevaine, ablette, hotu, brème bordelière, brème commune, carassin, carpe, Pseudorasbora parva, rotengle). 


\section{Tableau II}

Composition spécifique et amplitude des tailles des poissons passant par les pompes.

\section{Table II}

Species composition and size range of fish passing through the pumps.

\begin{tabular}{|c|c|c|c|c|c|c|}
\hline & & \multirow{2}{*}{$\begin{array}{l}\text { tailles capturées } \\
\text { (mini - maxi) }\end{array}$} & \multicolumn{2}{|c|}{ Sambuc } & \multicolumn{2}{|c|}{ Aube de Bouic } \\
\hline & & & effectif & $\%$ & effectif & $\%$ \\
\hline Gardon & Rutilus nutilus & $10-330$ & 1966 & 57,9 & 161 & 30,7 \\
\hline Brème bordelière & Blicca bjœrkna & $16-288$ & 236 & 7,0 & 75 & 14,3 \\
\hline Anguille & Anguilla anguilla & $58-463$ & 215 & 6,3 & 58 & 11,0 \\
\hline Goujon & Gobio gobio & $14-119$ & 278 & 8,2 & 46 & 8,8 \\
\hline Ablette & Alburnus alburnus & $15-126$ & 125 & 3,7 & 34 & 6,5 \\
\hline Carassin doré & Carassius auratus gibelio & $19-148$ & 18 & 0,5 & 47 & 9,0 \\
\hline Carpe & Cyprinus carpio & $18-60$ & 39 & 1,1 & 29 & 5,5 \\
\hline Hotu & Chondrostoma nasus & $22-132$ & 118 & 3,5 & 3 & 0,6 \\
\hline Chevaine & Leuciscus cephalus & $24-57$ & 89 & 2,6 & 8 & 1,5 \\
\hline Pseudorasbora & Pseudorasbora parva & $21-82$ & 61 & 1,8 & 11 & 2,1 \\
\hline Rotengle & Scardinius erythrophtalmus & $22-164$ & 32 & 0,9 & 14 & 2,7 \\
\hline Alose & Alosa fallax & $24-55$ & 20 & 0,6 & 14 & 2,7 \\
\hline Brème commune & Abramis brama & $25-86$ & 34 & 1,0 & 11 & 2,1 \\
\hline Perche soleil & Lepomis gibbosus & $31-109$ & 85 & 2,5 & 2 & 0,4 \\
\hline Barbeau & Barbus barbus & $25-135$ & 41 & 1,2 & 5 & 1,0 \\
\hline Sandre & Stizostedion lucioperca & $19-72$ & 11 & 0,3 & 3 & 0,6 \\
\hline Silure & Silurus glanis & $30-46$ & 1 & $<0,1$ & 4 & 0,8 \\
\hline Muges & Mugil spp. & $29-340$ & 10 & 0,3 & 0 & 0 \\
\hline Poisson chat & Ameinus melas & $109-192$ & 5 & 0,1 & 0 & 0 \\
\hline Loup & Dicentrarchus labrax & $22-48$ & 3 & 0,1 & 0 & 0 \\
\hline Loche franche & Neimacheilus barbatulus & $53-54$ & 2 & 0,1 & 0 & 0 \\
\hline Gremille & Gymnocephalus cernua & $33-96$ & 2 & 0,1 & 0 & 0 \\
\hline Bouviere & Rhodeus sericeus & 34 & 1 & $<0,1$ & 0 & 0 \\
\hline Tanche & Tinca tinca & 31 & 1 & $<0,1$ & 0 & 0 \\
\hline Gobie commun & Pomatoschistus microps & 10 & 1 & $<0,1$ & 0 & $\underline{0}$ \\
\hline
\end{tabular}

Vingt-quatre espèces ont été capturées au Sambuc, parmi lesquelles 16 ont été capturées à l'Aube de Bouic (tableau II). Les espèces dominantes numériquement sont les mêmes dans les deux stations : le gardon, l'anguille, le goujon et la brème bordelière. En plus des poissons, nous avons observé dans les deux stations des passages d'écrevisse américaine (Orconectes limosus), de gammare (Gammarus sp.) et de crevette d'eau douce (Atyaephyra desmaresti).

Le tableau III présente la distribution des captures des espèces dans les quatre compartiments du bassin du Fumemorte. Dans les données relatives aux canaux et au marais, les deux espèces de brèmes, bordelière et commune, sont rassemblées car elles n'ont pas été déterminées à l'espèce. Le premier axe de l'AFC de ce tableau, représentant $48 \%$ des variations, ordonne les compartiments de la manière suivante : le pompage, puis le canal d'irrigation, puis le canal de drainage et le marais, ces deux derniers compartiments étant très proches sur l'axe. Les espèces caractérisant le pompage et le canal d'irrigation sont le hotu, le loup, la loche franche, le chevaine, le barbeau. Le canal de drainage et le marais sont caractérisés par l'épinoche, le gobie commun, l'athérine et les muges.

\section{DISCUSSION}

A notre connaissance, il n'existe aucune autre étude publiée sur le passage de poissons par les pompes d'irrigation. Ce sujet est pourtant de première importance, 


\section{Tableau III}

Composition spécifique des différents compartiments du Bassin du Fumemorte (voir le texte pour le descriptif des stations). Les stations et les espèces sont ordonnées selon le premier axe de l'analyse factorielle des correspondances du tableau des espèces $x$ stations (F1), représentant $48 \%$ des variations. Les données des canaux d'irrigation et de drainage correspondent au même effort de pêche et sont donc comparables en valeurs absolues. Les captures de gambusie ne figurent pas dans ce tableau en raison de problèmes d'échantillonnage.

Table III

Species composition of different habitats in the Fumemorte catchment (see the text for the description of the sites). Sites and species are ordered by the first axis of the correspondence analysis of the species $x$ sites table (F1), representing $48 \%$ of the variations. Data from the irrigation and drainage canals correspond to the same sampling effort, so their raw values can be compared. Mosquitofish has been removed from this table because of sampling problems.

\begin{tabular}{|c|c|c|c|c|c|c|}
\hline Espèce & & Pompes & $\begin{array}{c}\text { Canal } \\
\text { d'irrigation }\end{array}$ & $\begin{array}{l}\text { Canal de } \\
\text { drainage }\end{array}$ & Marais & $\overline{F 1}$ \\
\hline Hotu & Chondrostoma nasus & 121 & & & & $2, \overline{97}$ \\
\hline Loche franche & Neimacheilus barbatulus & 2 & & & & 2,97 \\
\hline Loup & Dicentrarchus labrax & 3 & & & & 2,97 \\
\hline Chevaine & Leuciscus cephalus & 97 & 7 & & & 2,83 \\
\hline Barbeau & Barbus barbus & 46 & 3 & & 1 & 2,77 \\
\hline Gardon & Rutilus nutilus & 2127 & 160 & 123 & & 2,66 \\
\hline Goujon & Gobio gobio & 324 & 97 & 155 & & 1.72 \\
\hline Alose & Alosa fallax & 34 & 39 & 3 & 2 & 1.70 \\
\hline Grémille & Gymnocephalus cernua & 2 & 129 & 5 & & 0,85 \\
\hline Silure & Silunus glanis & 5 & 75 & 15 & 1 & 0.77 \\
\hline Carpe & Cyprinus carpio & 68 & 752 & 120 & 133 & 0,70 \\
\hline Perche & Perca fluviatilis & & 4 & 1 & & 0.62 \\
\hline Sandre & Stizostedion lucioperca & 14 & 23 & 55 & & 0,45 \\
\hline Carassin doré & Carassius auratus gibelio & 65 & 1 & 140 & 180 & 0,16 \\
\hline Brèmes & Blicca bjoerkna \& Abramis brama & 357 & 412 & 2703 & 1 & 0,13 \\
\hline Bouvière & Rhodeus senceus & 1 & 7 & 22 & 1 & 0.02 \\
\hline Perche soleil & Lepomis gibbosus & 87 & 89 & 995 & 61 & -0.04 \\
\hline Ablette & Alburnus alburnus & 159 & 115 & 1841 & 2 & $-0,04$ \\
\hline Anguille & Anguilla anguilla & 273 & 69 & 347 & 2301 & -0.11 \\
\hline Rotengle & Scardinius erythrophtalmus & 46 & 62 & 728 & 278 & $-0,18$ \\
\hline Brochet & Esox lucius & & 2 & 15 & 7 & $-0,29$ \\
\hline Pseudorasbora & Pseudorasbora parva & 72 & 331 & 5255 & 1509 & $-0,29$ \\
\hline Tanche & Tinca tinca & 1 & 1 & 31 & 15 & $-0,30$ \\
\hline Poisson chat & Ameirus melas & 5 & 77 & 1247 & 212 & $-0,30$ \\
\hline Muges & Mugil spp. & 10 & 3 & 1673 & 355 & $-0,36$ \\
\hline Spirlin & Alburnoides bipunctatus & & & 1 & & $-0,36$ \\
\hline Syngnathe & Syngnathus abaster & & & 5 & & $-0,36$ \\
\hline Athérine & Atherina boyer & & 1 & 8121 & 735 & -0.37 \\
\hline Gobie commun & Pomatoschistus microps & 1 & & 1397 & 676 & $-0,39$ \\
\hline Epinoche & Gasterosteus aculeatus & & & 941 & 3040 & $-0,44$ \\
\hline F1 & & 2.32 & 0,67 & $-0,28$ & $-0,36$ & \\
\hline Total des captures & & 3920 & 2459 & 25939 & 9510 & \\
\hline
\end{tabular}

notamment dans les zones poldérisées, pour lesquelles les pompes d'irrigation constituent l'unique possibilité de colonisation par les poissons. Dans le bassin du Fumemorte, en Camargue, les pompes d'irrigation constituent pratiquement la seule connexion avec tout autre milieu d'eau douce, le Rhône, et ainsi, l'unique possibilité pour des espèces strictement d'eau douce d'entrer dans l'île de Camargue.

Nos résultats ne permettent pas d'identifier une éventuelle mortalité des poissons causée par leur passage par les pompes d'irrigation. Dans les pompes des prises d'eau du 
circuit de refroidissement de centrales électriques, les estimations du taux de mortalité des poissons entrainés varient de $100 \%$ dans une pompe de 21 millions de l/s (MARCY, 1973), à 3 à $60 \%$ selon les espèces dans une pompe de $28000 \mathrm{l} / \mathrm{s}$ (VAN DENSEN et HADDERINGH, 1982). Cette mortalité est due à des chocs mécaniques, aux variations de pression, et à des chocs thermiques (MARCY, 1973). Ce type de pompes a des débits beaucoup plus élevés que les pompes d'irrigation étudiées ici, dont le débit maximum est de $1000 \mathrm{l} / \mathrm{s}$. De ce fait, les résultats relatifs aux pompes des centrales ne sont vraisemblablement pas applicables aux pompes d'irrigation. La présence d'individus de petite taille morts dans les pièges lorsque ceux-ci étaient chargés de nombreux poissons, nous apparaît comme une conséquence de l'écrasement au fond du piège, en raison du fort courant et de la résistance offerte par le piège, plutôt qu'une conséquence de leur passage dans la pompe. Ainsi, il nous semble que les poissons survivent au passage par la pompe.

Une première conséquence du passage de poissons du Rhône par les pompes d'irrigation est l'introduction d'espèces nouvelles en Camargue. Toutes les espèces nouvelles arrivées en Camargue ces dernières années (e.g., goujon, Pseudorasbora parva, grémille, silure, carassin doré, ROSECCHI et al., 1997) ont été observées dans le pompage. Toutefois, certaines de ces espèces peuvent avoir été aussi introduites en Camargue par d'autres biais, notamment lors de vidanges accidentelles de la pisciculture du Sambuc (ROSECCHI et al., 1997).

Nos résultats conduisent à estimer qu'en une saison rizicole, c'est-à-dire d'avril à octobre, environ 98000 individus entrent en Camargue par les pompes d'irrigation du bassin du Fumemorte. Cette estimation est grossière pour les raisons suivantes. Premièrement, la maille des pièges $(5 \mathrm{~mm}$ de vide de maille) ne permet pas de capturer efficacement les larves de poissons. Or, l'importance du phénomène de dérive chez les larves de poissons et leurs faibles capacités de nage les rendent particulièrement susceptibles d'être entraînés par les pompes (TURNPENNY et BAMBER, 1983 ; OLIVIER, 1992). Nos résultats sous-estiment donc fortement les quantités de poissons entrant par une pompe d'irrigation. Deuxièmement, notre estimation est grossière parce qu'elle correspond à une extrapolation à partir des données d'une seule station de pompage. L'influence de l'habitat sur la répartition des poissons dans le Rhône (POIZAT et PONT, 1996) doit certainement entraîner des différences entre pompes dans les quantités et la composition spécifique des poissons pompés, à la fois en fonction des caractéristiques locales de l'habitat dans lequel se situe la prise d'eau, et à une échelle plus large, en fonction de la distance entre la prise d'eau et l'embouchure du Rhône. Toutefois, les compositions spécifiques aux deux stations de pompage suivies présentent les mêmes espèces dominantes (tableau II). Enfin, il est probable que d'une année à l'autre les quantités de poissons pompées soient assez variables, en particulier pour le gardon et l'ablette qui montrent de fortes variations inter-annuelles dans leurs recrutements (POIZAT, 1993 ; CARREL et al., 1995). Malgré ces réserves, cette estimation fournit un ordre de grandeur des quantités de poissons introduites en Camargue par les pompes d'irrigation du bassin du Fumemorte.

Les peuplements des différents compartiments de l'hydrosystème de Camargue montrent un gradient " amont-aval " (tableau III). A l'amont, les pompes et le canal d'irrigation sont caractérisés par des espèces présentes dans le Rhône (POIZAT, 1993), mais qui n'ont pas de population établie en Camargue, telles que le hotu, le chevaine et le barbeau. Ces espèces ne sont maintenues en Camargue que par immigration via le pompage, mais elles ne s'y reproduisent pas, faute de sites de fraie adéquats. Elles se dispersent rarement au-delà du réseau d'irrigation. Par contre, pour l'alose qui se reproduit dans le Rhône, les juvéniles entrent en Camargue par le pompage et se dispersent dans les différents milieux de Camargue, y compris l'étang du Vaccarès, avant de migrer vers la mer dès les premiers froids (A.J. CRIVELLI et E. ROSECCHI, données non publiées). 
A l'aval, le canal de drainage et le marais sont caractérisés par des espèces réalisant des mouvements vers l'étang du Vaccarès et les étangs saumâtres inférieurs, telles que l'épinoche (CRIVELLI et BRITTON, 1987), l'athérine (ROSECCHI et CRIVELLI, 1995) et les muges (A.J. CRIVELLI et E. ROSECCHI, données non publiées).

La différenciation faunistique des canaux d'irrigation et de drainage peut s'expliquer par les connexions relativement réduites entre ces deux réseaux, qui se limitent d'une part aux milieux irrigués et drainés' tels que les rizières et les marais, et d'autre part aux surverses occasionnelles, liées au débordement de canaux d'irrigation se déversant directement dans le réseau de drainage (CHAUVELON, 1996). II est vraisemblable que les rizières ne soient que très peu utilisées par les poissons, car la'profondeur $y$ : est très faible (environ $10 \mathrm{~cm}$ ) et la température de l'eau peut y être très élevée $\left(25^{\circ} \mathrm{C}\right.$ en moyenne hebdomadaire, PONT, 1977). Donc, même si des poissons pouvaient entrer dans les rizières avec l'eau d'irrigation, il est peu probable qu'ils y survivent. L'eau des rizières s'écoule dans le réseau de drainage par une chute d'eau qui semble difficile à franchir par les poissons des canaux de drainage. Par contre, les marais constituent un passage plus plausible entre les réseaux d'irrigation et de drainage. Du point de vue physique, le passage de poissons du réseau d'irrigation au réseau de drainage est facilement réalisable par un marais qui serait irrigué puis drainé. Par contre, le passage du réseau de drainage au réseau d'irrigation est beaucoup plus difficile, car il implique qu'un marais, qui ait été déjà colonisé par les poissons via le réseau de drainage, soit à nouveau irrigué, et ainsi, que les poissons puissent remonter le courant vers le réseau d'irrigation. Les débordements des canaux d'irrigation peuvent permettre un passage direct des poissons vers le réseau de drainage, mais cette connexion ne permet pas les passages dans l'autre sens.

Ces contraintes physiques conduisent (1) à une dispersion facilitée de l'irrigation vers le drainage, expliquant pourquoi toutes les espèces abondantes dans le canal d'irrigation se retrouvent dans le canal de drainage et (2) à une difficulté de mouvements du drainage et des marais vers l'irrigation, expliquant pourquoi les espèces comme l'athérine, l'épinoche et le gobie commun sont absentes du réseau d'irrigation alors qu'elles sont abondantes dans le réseau de drainage et les marais.

Le peuplement du marais est proche de celui du canal de drainage, mais il s'en distingue par l'absence d'espèces telles que les brèmes, le gardon, le goujon et le sandre. Une étude antérieure sur les peuplements d'autres marais temporaires de Camargue (POIZAT et CRIVELLI, 1997), a montré des résultats similaires pour ces espèces qui ne fréquentent pas les marais bien qu'elles en aient l'accès. En dehors des facteurs physiques liés à la gestion hydraulique, le fait que le peuplement du marais ressemble davantage au canal de drainage qu'au canal d'irrigation peut aussi s'expliquer par la densité de poissons du canal de drainage qui est de l'ordre de 10 fois supérieure à celle du canal d'irrigation (tableau III), ceci augmentant les possibilités de colonisation par le drainage.

Pour les espèces dont les populations en place dans le bassin du Fumemorte sont importantes, l'importance de l'immigration par pompage dans le fonctionnement des populations reste difficile à apprécier car on ne connaît pas la taille de leurs populations en Camargue. Pour l'anguille, qui se reproduit en mer, il faudrait connaître l'importance des entrées par le grau de la Fourcade et le grau de Rousty pour apprécier l'importance relative des entrées par pompage dans son recrutement en Camargue.

\section{REMERCIEMENTS}

Nous remercions les Syndicats de gestion de l'Aube de Bouic et du canal du Sambuc d'avoir accepté que l'on réalise ces suivis, Christophe PAMPOULIE pour la détermination 
des gobies, et François PRIOUR, Elodie GAZAVE et Sylviane CHOSSON pour leur participation au travail de terrain. Ce travail a été financé par la Fondation Tour du Valat.

\section{BIBLIOGRAPHIE}

BARDIN O., 1994. Facteurs régulant les échanges piscicoles entre la mer et les étangs centraux de Camargue. Rapport de DEA, Université Aix-Marseille III, 31 p.

CARREL G., PONT D., RIVIER B., 1995. Variabilité temporelle des peuplements piscicoles dans la section médiane du Bas-Rhône. Bull. Fr. Pêche Piscic., 337/338/339, 101111.

CHAUVELON P., 1996. Hydrologie quantitative d'une zone humide méditerranéenne aménagée : le bassin du Fumemorte en Grande Camargue, delta du Rhône. Thèse de Doctorat, Université Montpellier II, $275 \mathrm{p}$.

CHAUVELON P., 1998. A wetland managed for agriculture as an interface between the Rhone River and the Vaccarès lagoon (Camargue, France) : transfers of water nutrients. Hydrobiologia, 373/374, 181-191.

CHAUVELON P., SANDOZ A., POIZAT G., CRIVELLI A.J., 1996. Hydro-agricultural management in the Rhone River delta, France : consequences on dissolved and solid fluxes, potential impact on fish populations. Proceedings of the second IAHR Symposium on Habitat Hydraulics, Quebec, June 1996, 167-176.

CRIVELLI A.J., 1981. Les peuplements de poissons de la Camargue. Rev. Ecol. Terre Vie, 35, 617-671.

CRIVELLI A.J., BRITTON R.H., 1987. Life history adaptations of Gasterosteus aculeatus in a Mediterranean wetland. Env. Biol. Fishes, 18, 109-125.

CRIVELLI A.J., MESTRE D., 1988. Life history traits of pumpkinseed, Lepomis gibbosus, introduced into the Camargue, a Mediterranean wetland. Arch. Hydrobiol., 111, 449466.

HEURTEAUX P., 1994. Essai de quantification des termes du bilan hydrique des étangs du système Vaccarès (Camargue, France). Ann. Limnol., 30, 131-134.

MARCY B.C., Jr., 1973. Vulnerability and survival of young Connecticut River fish entrained at a nuclear power plant. J. Fish. Res. Board Can., 30, 1195-1203.

OLIVIER J.M., 1992. Rythmes de dérive des alevins en milieu fluvial. Suivi dans le Rhône au niveau des prises d'eau et influence des vidanges de barrages. Thèse de Doctorat, Université Lyon I, 129 p. + figures et annexes.

POIZAT G., 1993. Echelle d'observation et variabilité des abondances de juvéniles de poissons dans un secteur aval du Rhône. Thèse de Doctorat, Université Lyon I, $217 \mathrm{p}$.

POIZAT G., PONT D., 1996. Multi-scale approach to species-habitat relationships : juvenile fish in a large river section. Freshwater Biol., 36, 611-622.

POIZAT G., CRIVELLI A.J., 1997. Use of seasonally flooded marshes by fish in a Mediterranean wetland : timing and demographic consequences. J. Fish. Biol., 51, 106-119.

PONT D., 1977. Structure et évolution saisonnière des populations de copépodes, cladocères et ostracodes des rizières de Camargue. Annls Limnol., 13, 15-28.

ROSECCHI E., CRIVELLI A.J., 1992. Study of a sand smelt (Atherina boyeri Risso, 1810) population reproducing in freshwater. Ecology of Freshwater Fish, 1, 77-85.

ROSECCHI E., CRIVELLI A.J., 1995. Sand smelt (Atherina boyeri) migration within the water system of the Camargue, southern France. Hydrobiologia, 300/301, 289-298. 
ROSECCHI E., POIZAT G., CRIVELLI A.J., 1997. Introductions de poissons d'eau douce et d'écrevisses en Camargue : historique, origines et modifications des peuplements. Bull. Fr. Pêche Piscic., 344/345, 221-232.

THIOULOUSE J., CHESSEL D., DOLEDEC S., OLIVIER J.M., 1997. ADE-4 : a multivariate analysis and graphical display software. Statistics Computing, 7, 75-83.

TURNPENNY A.W.H., BAMBER R.N., 1983. The critical swimming speed of the sand smelt (Atherina presbyter Cuvier) in relation to capture at a power station cooling water intake. J. Fish. Biol., 23, 65-73.

VAN DENSEN W.L.T., HADDERINGH R.H., 1982. Effects of entrapment and cooling water discharge by the Bergum Power Station on $0+$ fish in. the Bergumermeer. Hydrobiologia, 95, 351-368. 